\title{
Measuring the Proton Radius in High-Energy Muon-Proton Scattering
}

\section{Christian Dreisbach ${ }^{* a}$, Jan M. Friedrich ${ }^{a}$, Martin Hoffmann ${ }^{b}$, Alexander Inglessi ${ }^{c}$, Eva Kabuß $^{d}$, Bernhard Ketzer ${ }^{b}$, Oleg Kiselev ${ }^{e}$ Evgeny Maev $^{c}$, Stephan Paul ${ }^{a}$, Gennady Petrov ${ }^{c}$, Sebastian Uhl ${ }^{a}$, Alexander Vasilyev ${ }^{c}$, Benjamin M. Veit ${ }^{d, f}$, Alexey A. Vorobyov ${ }^{c}$ and the COMPASS++/AMBER working group $f$}

${ }^{a}$ Technical University of Munich, Department of Physics

${ }^{b}$ University of Bonn, Helmholtz-Institut für Strahlen- und Kernphysik

${ }^{c}$ Petersburg Nuclear Physics Institute (PNPI) of the National Research Centre, Kurchatov Institute

${ }^{d}$ Johannes Gutenberg University Mainz, Institute for Nuclear Physics

${ }^{e}$ GSI Helmholtzzentrum für Schwerionenforschung

${ }^{f}$ European Organization for Nuclear Research (CERN)

E-mail: christian.dreisbach@cern.ch

\begin{abstract}
The proton charge radius can be determined by measuring the slope of the electric form-factor $G_{E}$ at small four-momentum transfer squared $Q^{2}$. Numerous elastic-scattering and laser-spectroscopy measurements of the proton radius have been performed with contradicting results, often referred to as the proton-radius puzzle. We propose to measure the proton charge radius in high-energy elastic muon-proton scattering at the M2 beam line of CERN's Super Proton Synchrotron (SPS) in 2022. A high-precision measurement at low $Q^{2}$, performed with a high-pressure hydrogen-filled time-projection chamber (TPC), can contribute to the resolution of the puzzle, especially due to the different systematic effects of this approach compared to those of electron-proton scattering. In 2018, we performed a test measurement with silicon tracking detectors up- and downstream of a prototype TPC to study the feasibility of the measurement concept. We present initial results of the on-going analysis of the test data and discuss ideas for a possible experiment at CERN in 2022.
\end{abstract}

XXVII International Workshop on Deep-Inelastic Scattering and Related Subjects - DIS2019 8-12 April, 2019

Torino, Italy

* Speaker. 


\section{Introduction}

The persistent discrepancy between the proton charge radius measured in muonic or ordinary hydrogen spectroscopy and electron scattering experiments is about 5.6 $\sigma$. In addition, various models applied to the data yield to different values of the extracted proton charge radius. Therefore, we propose to measure the proton charge radius in high-energy muon-proton elastic scattering over a wide $Q^{2}$-range of $0.001 \leq Q^{2} /\left(\mathrm{GeV}^{2} / c^{2}\right) \leq 0.04$ at CERN's M2 beam line located at the SPS in 2022 using the experimental setup partially basing on the existing COMPASS spectrometer to test the stability of possible fit models over the measured $Q^{2}$-range and to contribute with a high-energy muon scattering to the proton radius puzzle [1].

The proton charge radius is defined by the slope of the electric form-factor $G_{E}$ as given in Eq. 1.1.

$$
<r_{E}^{2}>=-\left.6 \hbar^{2} \cdot \frac{d G_{E}\left(Q^{2}\right)}{d Q^{2}}\right|_{Q^{2} \rightarrow 0}
$$

The electric form-factor $\mathrm{G}_{E}$ can be accessed by measuring the differential cross-section of the elastic muon-proton scattering given in the Rosenbluth separation in Eq. 1.2.

$$
\begin{gathered}
\frac{d \sigma^{\mu p \rightarrow \mu^{\prime} p}}{d Q^{2}}=\frac{4 \pi \alpha^{2}}{Q^{4}} R\left(\varepsilon G_{E}^{2}+\tau G_{M}^{2}\right) \quad \text { with } \\
R=\frac{{\overrightarrow{p_{\mu}}}^{2}-\tau\left(s-2 m_{p}^{2}(1+\tau)\right)}{{\overrightarrow{p_{\mu}}}^{2}(1+\tau)} \quad, \quad \varepsilon=\frac{E_{\mu}^{2}-\tau\left(s-m_{\mu}^{2}\right)}{{\overrightarrow{p_{\mu}}}^{2}-\tau\left(s-2 m_{p}^{2}(1+\tau)\right)} \quad \text { and } \quad \tau=\frac{Q^{2}}{4 m_{P}^{2}}
\end{gathered}
$$

Using the center-of-mass energy $s=2 E_{\mu} m_{p}+m_{p}^{2}+m_{\mu}^{2}$ in the laboratory frame and the incoming muon colliding with a proton at rest. For small $Q^{2}$ the contribution of the magnetic form-factor $G_{M}^{2}$ is suppressed $(\tau \rightarrow 0)$ and for high beam energies $E_{\mu}>100 \mathrm{GeV} / c$ the cross-section is directly proportional to the electric form-factor $\mathrm{G}_{E}^{2}\left(\varepsilon \rightarrow 1\right.$ ). In Fig. 1 (top) the proton form-factors $\mathrm{G}_{E}$ and $\mathrm{G}_{M}$ as measured at MAMI [2] are shown relative to the dipole form-factor $\mathrm{G}_{D}$ together with the resulting slopes for the electric form factor $\mathrm{G}_{E}$ of different proton radii extracted by muonic hydrogen spectroscopy and elastic electron-proton scattering.

We plan to collect about $10^{8}$ events, which then will constrain the charge radius of the proton with precision better than $0.01 \mathrm{fm}$. The statistical uncertainties are expected to dominate over the systematic point-to-point uncertainty needed to estimate the slope of the electric form-factor. A deviation from a point-like proton at the lowest proposed $Q^{2}$-value is expected to be on the $10^{-3}$ level, which is smaller than unavoidable systematic effects like detector efficiencies. In Fig. 1 (bottom) the cross-section behavior extracted by MAMI relative to the standard dipole form-factor is shown. Data will be available from the proposed experiment down to $Q^{2} \leq 0.001 \mathrm{GeV}^{2} / c^{2}$, with the statistical uncertainty further shrinking according to the increasing cross-section with $Q^{2} \rightarrow 0$.

\section{Proton-radius measurement based on the COMPASS spectrometer}

For the challenge of a measurement in the low- $Q^{2}$ region a Time-Projection Chamber (TPC) is planned to be used as an active target for detection of low-energetic recoil-protons stemming from 

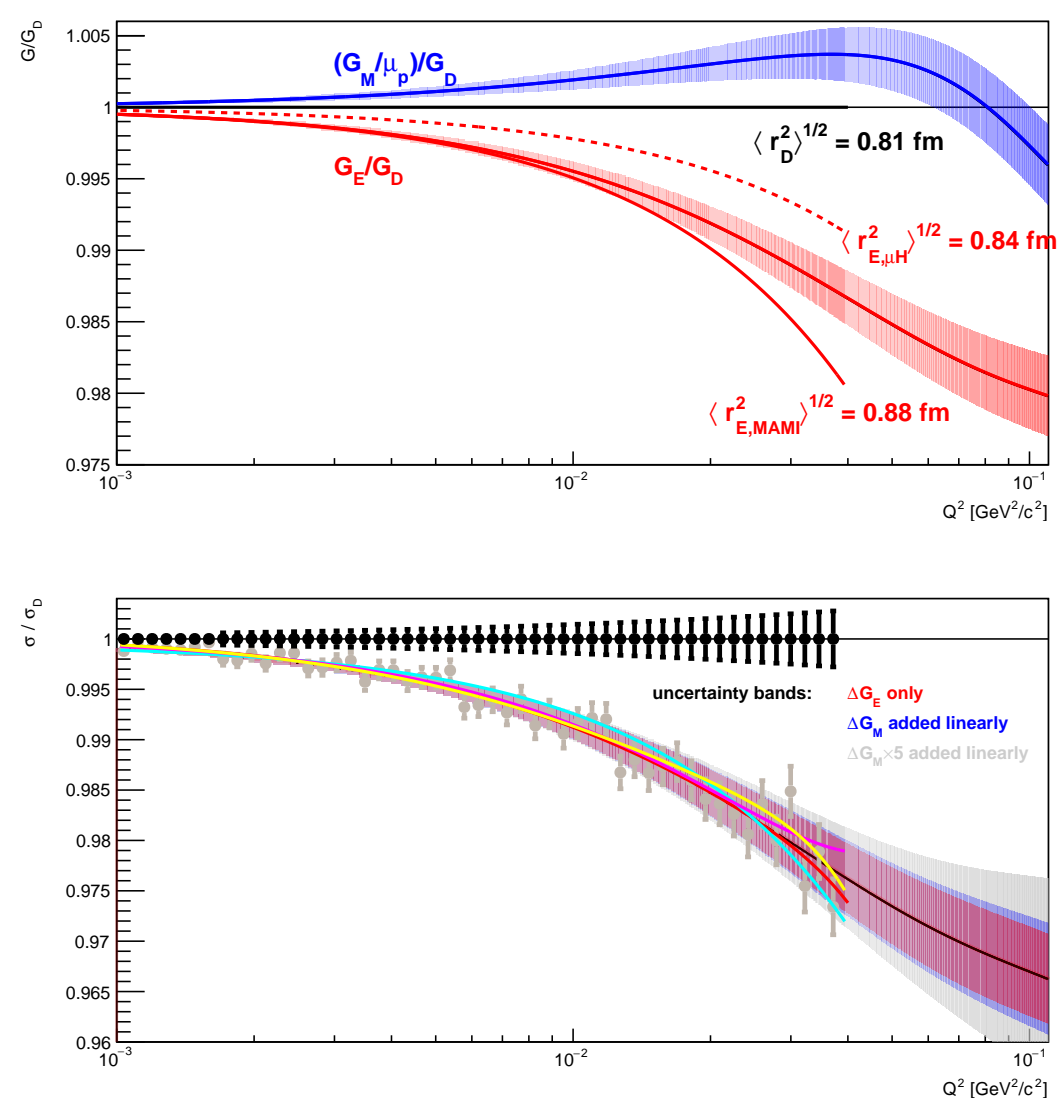

Figure 1: Top: Proton form-factors $\mathrm{G}_{E}$ and $\mathrm{G}_{M}$ as measured at MAMI, presented relative to the dipole form $\mathrm{G}_{D}$. Bottom: Corresponding cross-section behavior relative to the standard dipole-form with uncertainty contributions of $\Delta \mathrm{G}_{E}$ and $\Delta \mathrm{G}_{M}$. The dots with error bars, arbitrarily placed at 1, represent the achievable statistical precision of the proposed measurement. [1]

the elastic muon-proton scattering. The measurement of small scattering-angles of the deflected muons requires silicon detectors for precise vertexing and low material-budget to reduce the influence of multiple scattering. For the selection of elastic events a fiber trigger array is planned. Vacuum or helium tubes will be used for further reduction of multiple scattering along the baselines of the setup. A possible layout of the proposed setup is shown in Fig. 2. With an overall baseline of $11 \mathrm{~m}$ a resulting leaver arm of $5 \mathrm{~m}$ is achieved to obtain the required angular precision of less than $100 \mu \mathrm{rad}$ to measure $Q^{2}$-values down to $\mathscr{O}\left(10^{-3}\right)$.

The proposed measurement can take place at CERN's M2 beam line providing a unique muon beam with a momentum of $100 \mathrm{GeV} / \mathrm{c}$ and a maximal intensity of $5.6 \cdot 10^{7} \mu / \mathrm{s}$. With an integrated cross-section for the elastic muon-proton scattering of 0.26 mbarn at this beam energy and $0.001 \leq$ $Q^{2} /\left(\mathrm{GeV}^{2} / c^{2}\right) \leq 0.04$ about $10^{8}$ events are required for sufficient statistics to extract the proton charge radius with the proposed precision. The measurement over this wide $Q^{2}$-range needs to be split into two sub-measurements with different TPC pressure settings of 4 bar and 20 bar resulting in 140 days of data taking.

For the measurement of the proton charge radius using the modified COMPASS spectrometer a TPC, silicon detectors and a fiber trigger array as new key detector elements need to be developed. 


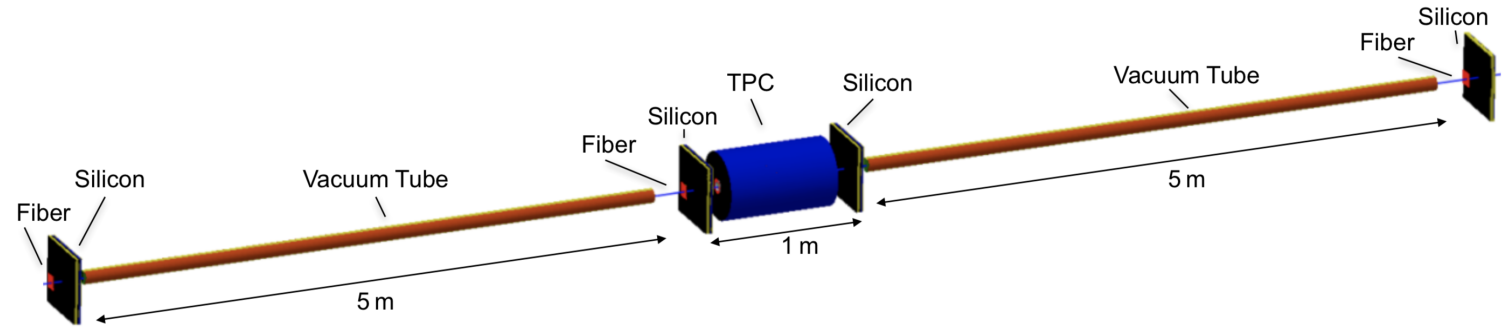

Figure 2: Layout of the $11 \mathrm{~m}$ long target region consisting of an active target TPC, silicon detectors for track reconstruction, fiber trigger used for the selection of deflected muons and vacuum tubes to minimize the multiple scattering effect.

Currently the MuPix8 [3] prototype is under investigation as a candidate for the silicon detectors. In the current design it has a size of $1 \times 2 \mathrm{~cm}^{2}$ consisting of $128 \times 200$ pixels each with a size of 80x80 $\mu \mathrm{m}^{2}$. The integrated read-out-system and its capability to be thinned down to only $50 \mu \mathrm{m}$ are an advantage in terms of multiple scattering. A possible large-area design with sizes larger than $4 \times 4 \mathrm{~cm}^{2}$ is under investigation. For the final setup four stations consisting of up to four detector layers are planned. The fiber trigger stations will consist of $200 \mu \mathrm{m}$ thick fibers grouped in four layers. Each fiber will be read out by a dedicated SiPMT. Three fiber trigger stations are combined to a trigger system to detect deflected muons. A TPC filled with pressurized hydrogen will be used as an active target. The pressure can vary up to 20 bar, which enables the measurement of the recoil proton over the wide $Q^{2}$-range with recoil-proton energies from $0.5 \mathrm{MeV}$ to $20 \mathrm{MeV}$.

Measurements at low momentum-transfer and resulting small scattering-angles are heavily effected by multiple scattering. For an estimate the material budget of the contributing parts of the inner target area are taken into account. The material of two silicon stations, one fiber trigger station and the TPC contribute. An overview of the material contribution is show in Fig. 3 (left). This configuration makes use of helium filled tubes between the silicon stations of each telescope arm. The main contribution to the material budget is produced by silicon followed by beryllium of the TPC windows. Remaining contribution is due to other materials of the TPC and the fiber stations. The effect of multiple scattering on the measured $Q^{2}$ is shown in Fig. 3 (right) for different beam energy scenarios. For the TPC a maximal $Q^{2}$-value limits the detectable recoil protons inside the detector. The tracking minima and maxima result from the geometry, sizes and respective spatial resolutions of the tracking detectors.

\section{Preceding test measurement}

As preparation for the proposed measurement in 2022 a test measurement was performed in 2018 with the setup located downstream of the COMPASS spectrometer during the 2018 Drell-Yan run using the remaining muons of the $190 \mathrm{GeV} / \mathrm{c}$ pion beam. The goal was to study the feasibility of such a setup consisting of a TPC and silicon detectors. Beam-rate studies, especially with the broad beam were performed together with collecting overall experience for the operation of a comparable future measurement. The test setup consisted of two independent detector types with two dedicated DAQ systems. Using a TPC as a slow detector combined with comparable fast silicon detectors upand downstream of the TPC required a later event matching using a so-called TRLO timestamp. 

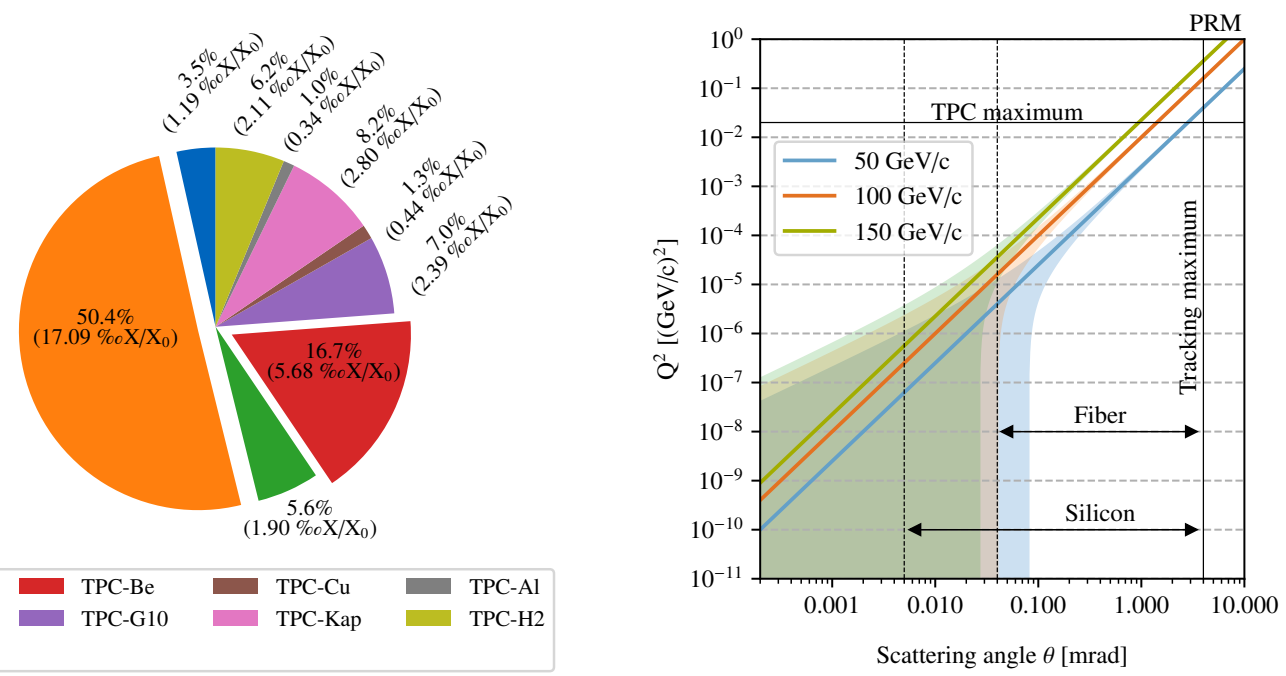

Figure 3: Left: Material budget of the proposed setup. For each material the contribution to the total radiation length is given. Right: Different beam energy scenarios with their uncertainty bands due to multiple scattering are shown. The kinematic region is limited by the maximal momentum of the recoil proton detectable via the TPC, the geometry and resolution of the setup and tracking detectors respectively.

The ACTAF2/R3B prototype TPC was used with its $230 \mathrm{~mm}$ long drift volume defined by the anode containing the read-out plane and the cathode. Since the ACTAF2 was designed and operated in 2017 at MAMI's pencil-like electron beam the read-out plane is structured into segmented rings each with 16 pads. Figure 4 (left and center) shows a schematic drawing of the TPC and its read-out plane. In Fig. 5 (left and center) the primary vertex distributions in $x y$ - and $z$-direction are shown. Along the $2 \mathrm{~m}$ long target area the single components of the setup are visible. The silicon stations SI02 and SI03 are located up- and downstream of the TPC. Single components of the TPC such as the up- and downstream entrance windows together with its anode and cathode represent the inner structure. Using the time stamp generated by the DAQ systems the time difference for each event can be used to match possible candidates. The resulting time difference between TPC and silicon detector events is shown in Fig. 5 (right). The location is slightly shifted due to latency and its width corresponds to the overall drift time between anode and cathode. The selected events within the drift time window are in agreement with recoil proton candidates independently measured by the TPC shown in Fig. 4 (right). In addition, other kinematic variables like the polar angle and recoil energy are consistent between both measurements [4].

\section{Conclusion}

The performed tests demonstrated the possibility of sound event matching between two distinct DAQ systems for a TPC and silicon detectors via time stamp. Nevertheless, a reasonable vertex resolution is crucial to identify scattering in the hydrogen gas of the TPC. For a future and much more precise measurement silicon detectors, large-volume TPC and fiber detectors as trigger on the deflected muon are currently developed taking the important effect of multiple scattering into 

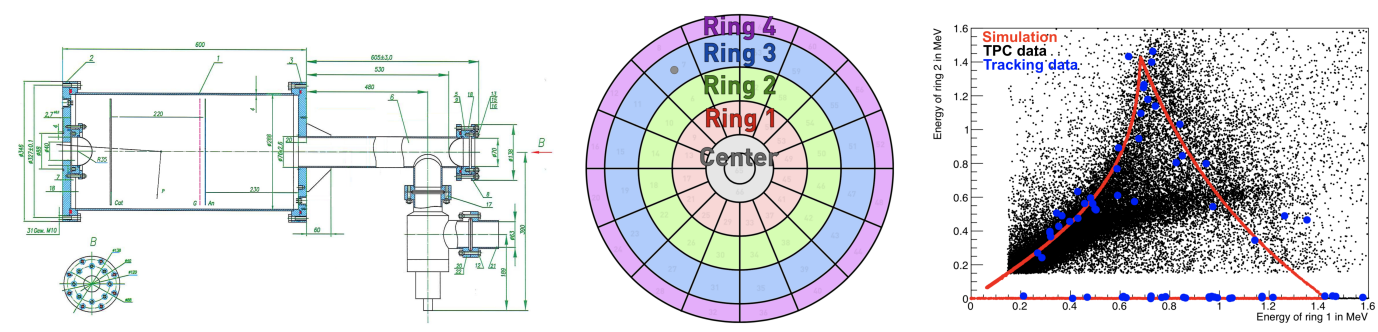

Figure 4: Left: Schematic drawing of the prototype TPC for the test setup with the active volume located between anode and cathode. Center: The segmented ring structure is located on the anode side. Right: A correlation between recoil protons directly measured in the TPC and reconstructed by the silicon detectors is visible.
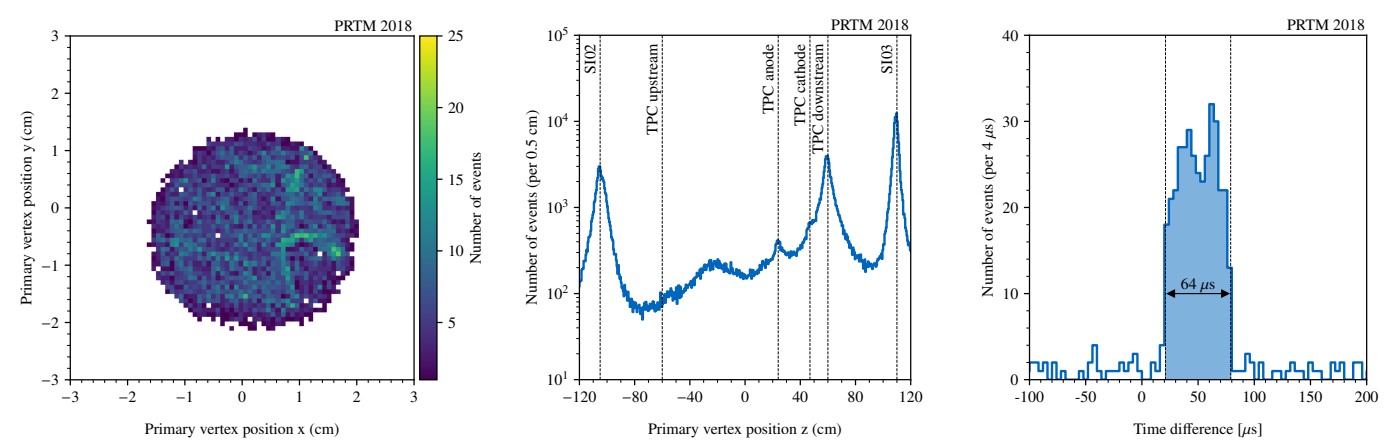

Figure 5: Left and center: Vertex distribution along $x y$ - and z-direction. Cabling is visible in $x y$-plane, as well as single components of the setup along the $z$-axis. Right: Time difference of both DAQ events of the test measurement. The resulting time difference is in agreement with the maximal drift time of the TPC.

account. In addition, studies on the overall layout of a modified COMPASS spectrometer, DAQ system and required beam properties are ongoing.

The proposed measurement can take place at CERN's unique M2 beam line. For the targeted precision of $0.01 \mathrm{fm}$ for the proton charge radius about $10^{8}$ events are required resulting in roughly 140 days of data taking with a accurate stability control of all crucial environment variables.

\section{References}

[1] Adams et al., Compass++/AMber: Proposal for Measurements at the M2 beam line of the CERN SPS Phase-1: 2022-2024, CERN-SPSC-2019-022. SPSC-P-360", May 2019

[2] J. C. Bernauer et al., High-precision determination of the electric and magnetic form factors of the proton, Phys. Rev. Lett.105 (2010) 242001

[3] H. Augustin et al., Mupix8 large area monolithic hvcmos pixel detector for the mu3e experiment, Nuclear Instruments and Methods in Physics Research Section A: Accelerators, Spectrometers, Detectors and Associated Equipment

[4] M. Hoffmann, A feasibility test for measuring the proton charge radius in high-energy muon-proton elastic scattering, Master thesis, Helmholtz-Institut für Strahlen- und Kernphysik, Universität Bonn, May 2019 